\title{
Carrier Frequency Offsets Problem in DCT-SC-FDMA System: Investigation and Compensation
}

\author{
Faisal S. Al-kamali, Moawad I. Dessouky, Bassiouny M. Sallam, Farid Shawki, \\ and Fathi E. Abd El-Samie \\ Department of Electronics and Electrical Communications, Faculty of Electronic Engineering, Menoufia University, \\ Menouf 32952, Egypt \\ Correspondence should be addressed to Faisal S. Al-kamali, faisalalkamali@yahoo.com
}

Received 10 August 2010; Accepted 2 September 2010

Academic Editors: R. Pérez Jiménez and A. B. Sesay

Copyright (๑) 2011 Faisal S. Al-kamali et al. This is an open access article distributed under the Creative Commons Attribution License, which permits unrestricted use, distribution, and reproduction in any medium, provided the original work is properly cited.

\begin{abstract}
The Single-Carrier Frequency Division Multiple Access (SC-FDMA) system is a well-known system, which has recently become a preferred choice for uplink channels. In this system, the Carrier Frequency Offsets (CFOs) disrupt the orthogonality between subcarriers and give rise to Intercarrier Interference (ICI), and Multiple Access Interference (MAI) among users. In this paper, the impact of the CFOs on the performance of the Discrete Cosine Transform (DCT) SC-FDMA (DCT-SC-FDMA) system is investigated. Then, a new low-complexity joint equalization and CFOs compensation scheme is proposed to cancel the interference in frequency domain. The Minimum Mean Square Error (MMSE) equalizer is utilized in the proposed scheme. A hybrid scheme comprising MMSE equalization, CFOs compensation, and Parallel Interference Cancellation (PIC) is also suggested and investigated for further enhancement of the performance of the DCT-SC-FDMA system with interleaved subcarriers assignment. For simplicity, this scheme will be referred to as the MMSE+PIC scheme. From the obtained simulation results, it is found that the proposed schemes are able to enhance the system performance, even in the presence of the estimation errors.
\end{abstract}

\section{Introduction}

In the SC-FDMA system, each user employs a different set of orthogonal subcarriers as in the Orthogonal Frequency Division Multiple Access (OFDMA) system. For perfect time and frequency synchronization, the different orthogonal subcarriers sets for the different users make it possible to avoid the MAI $[1,2]$. However, similar to the OFDMA system, the SC-FDMA system is sensitive to CFOs, which are mainly due to oscillator mismatches and/or Doppler shifts. As a result, in this system, the CFOs disrupt the orthogonality between subcarriers and give rise to ICI and MAI among users [3, 4]. Moreover, CFOs compensation is difficult in uplink communications since the CFOs compensation for a certain user may result in the misalignment of the other synchronized users [4].

The existing SC-FDMA system uses the Discrete Fourier Transform (DFT) in its implementation. A method for using the DCT as an alternative to the DFT is presented in [5], where we have introduced an improved DCT-SC-FDMA system and compared its performance with that of the DFTSC-FDMA and the OFDMA systems.

The issue of CFOs in multicarrier systems was extensively studied in the literature [6-10]. However, for the uplink SC-FDMA system, there have been a few papers that addressed this issue $[3,4,11,12]$. In [3], the impact of the CFOs compensation on the performance of Multiple-Input Multiple-Output (MIMO) DFT-SC-FDMA system was investigated. Frequency offsets estimation for high-speed users in E-UTRA uplink was proposed and investigated in [4]. In [11], the authors proposed an equalizer to mitigate the impact of the residual MAI after the CFOs compensation process in the DFT-SC-FDMA system. A joint suppression method for the phase noise and CFOs by block-type pilots for the MIMO DFT-SC-FDMA system was discussed in [12]. 


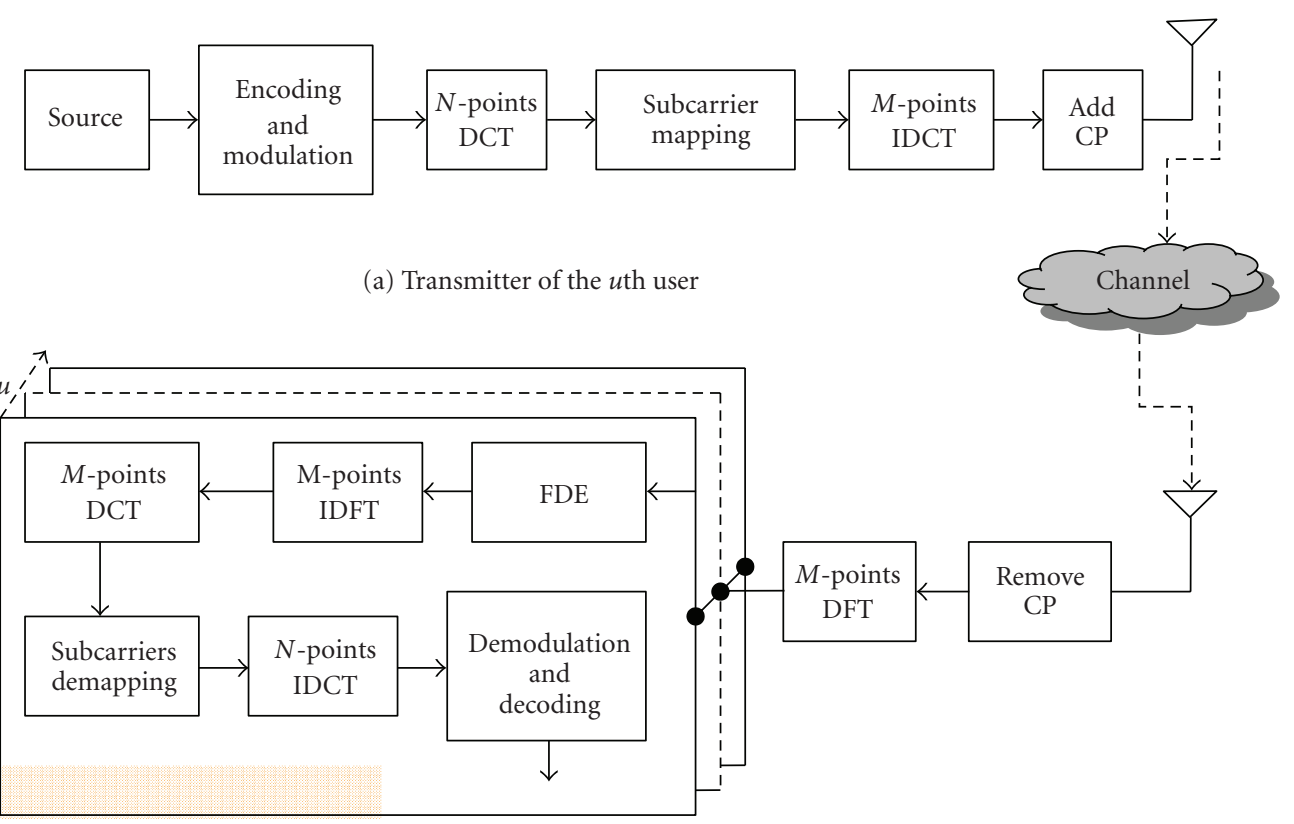

(b) Receiver (base station)

FIgURE 1: Structure of the DCT-SC-FDMA system.

Up to now, the impact of CFOs on the performance of the DCT-SC-FDMA system has not been investigated. This impact and how to reduce it is the main objective of this paper. Moreover, to the best of our knowledge, no work has been reported on the joint equalization and CFOs compensation for uplink systems. This motivated us to do the research work in this paper. Two types of the DCT-SC-FDMA system are studied: the system with interleaved subcarriers assignment which will be referred to as the DCT-IFDMA system and the system with localized subcarriers assignment which will be referred to as the DCT-LFDMA system. The rest of the paper is organized as follows. In Section 2, we introduce the system model for the uplink DCT-SC-FDMA system in the presence of CFOs. Also in Section 3, the proposed joint equalization and CFOs compensation scheme is presented. We also present a lowcomplexity implementation of this scheme using a banded matrix approximation. In Section 3, the computational complexity of the proposed equalization scheme is investigated. The algorithm of the suggested MMSE + PIC scheme is introduced in Section 4. The simulation results are presented in Section 5, and the conclusions are given in Section 6.

Throughout this paper, the symbols $(\cdot)^{H},(\cdot)^{T},(\cdot)^{*}$ and $(\cdot)^{-1}$ denote complex conjugate transposition of a matrix, transposition of a matrix, complex conjugate of a matrix, and the inverse of a matrix, respectively. Vectors and matrices are represented in boldface.

\section{DCT-SC-FDMA System Model}

This section describes the uplink DCT-SC-FDMA system. We consider $U$ users communicating at the same time with a fixed base station through independent multipath Rayleigh-fading channels as shown in Figure 1. The received signals from all users at the base station are assumed to be synchronized in the time domain.

At the transmitter side, the modulated symbols are grouped into blocks and an $\mathrm{N}$-points DCT is performed. Then, the subcarriers are mapped in the frequency domain. After that, an $M$-points Inverse DCT (IDCT) is performed and a Cyclic Prefix (CP) of length $N_{C}$ is added to the resulting signal. The length of the $\mathrm{CP}$ must be greater than the maximum excess delay of the channel to accommodate for the Interblock Interference (IBI). Finally, the resulting signal is transmitted through the wireless channel.

In matrix notation, the transmitted signal from the $u$ th user $(u=1,2, \ldots, U)$ can be formulated as follows:

$$
\overline{\mathbf{x}}^{u}=\mathbf{P}_{\mathrm{add}} \mathbf{D}_{M}^{-1} \mathbf{M}_{T}^{u} \mathbf{D}_{N} \mathbf{x}^{u},
$$

where $\mathbf{x}^{u}$ is an $N \times 1$ vector containing the modulated symbols. $\mathbf{D}_{N}$ is the $N \times N$ DCT matrix. $\mathbf{M}_{T}^{u}$ is an $M \times N$ matrix describing the subcarriers mapping of the $u$ th user. $\mathbf{D}_{M}^{-1}$ is the $M \times M$ IDCT matrix. $M=Q \cdot N$, where $Q$ is the maximum number of users that can transmit, simultaneously. $\mathbf{P}_{\text {add }}$ is an $\left(M+N_{C}\right) \times M$ matrix, which adds a CP of length $N_{C}$. The entries of $\mathbf{M}_{T}^{u}$ for both DCT-LFDMA and DCT-IFDMA are given, respectively, in

$$
\begin{gathered}
\mathbf{M}_{T}^{u}=\left[\mathbf{0}_{(u-1) N \times N} ; \mathbf{I}_{N} ; \mathbf{0}_{(M-u N) \times N}\right], \\
\mathbf{M}_{T}^{u}=\left[\mathbf{0}_{(u-1) \times N} ; \mathbf{u}_{1}^{T} ; \mathbf{0}_{(Q-u) \times N} ; \ldots ; \mathbf{0}_{(u-1) \times N} ; \mathbf{u}_{N}^{T} ; \mathbf{0}_{(Q-u) \times N}\right],
\end{gathered}
$$

where the $\mathbf{I}_{N}$ and $\mathbf{0}_{Q^{\prime} \times N}$ matrices denote the $N \times N$ identity matrix and the $Q^{\prime} \times N$ all-zero matrix, respectively. 
$\mathbf{u}_{n}$ denotes the unit column vector, of length $N$, with all zero entries except at $n$. $\mathbf{P}_{\text {add }}$ can be represented as follows:

$$
\mathbf{P}_{\mathrm{add}}=\left[\mathbf{C}, \mathbf{I}_{M}\right]^{T},
$$

where

$$
\mathbf{C}=\left[\mathbf{0}_{N_{C} \times\left(M-N_{C}\right)}, \mathbf{I}_{N_{C}}\right]^{T} .
$$

Assuming perfect time synchronization at the receiver side, the received signal can be expressed as follows:

$$
\overline{\mathbf{r}}=\sum_{u=1}^{U} \overline{\mathbf{E}}^{u} \mathbf{H}^{u} \overline{\mathbf{x}}^{u}+\mathbf{n},
$$

where $\overline{\mathbf{E}}^{u}$ is an $\left(M+N_{C}\right) \times\left(M+N_{C}\right)$ diagonal matrix with elements $\left[\overline{\mathbf{E}}^{u}\right]_{m, m}=e^{j \pi \zeta_{u}(2 m+1) / 2 M}, m=0, \ldots, M+N_{C}-1$, which describes the CFO matrix of the $u$ th user for the DCTSC-FDMA system. $\zeta_{u}$ is the normalized CFO of the $u$ th user with respect to the subcarriers frequency spacing. $\mathbf{H}^{u}$ is an $\left(M+N_{C}\right) \times\left(M+N_{C}\right)$ matrix describing the channel of the $u$ th user. $\mathbf{n}$ is an $\left(M+N_{C}\right) \times 1$ vector containing the noise. After the removal of the $\mathrm{CP}$, the received signal becomes

$$
\mathbf{r}=\mathbf{P}_{\mathrm{rem}} \overline{\mathbf{r}}=\sum_{u=1}^{U} \mathbf{E}^{u} \mathbf{H}_{C}^{u} \widetilde{\mathbf{x}}^{u}+\widetilde{\mathbf{n}},
$$

where $\mathbf{P}_{\text {rem }}$ is an $M \times\left(M+N_{C}\right)$ matrix, which removes the $\mathrm{CP}$. It is given by

$$
\mathbf{P}_{\mathrm{rem}}=\left[\mathbf{0}_{\left(M \times N_{C}\right)}, \mathbf{I}_{M}\right] .
$$

$\mathrm{E}^{u}$ is an $M \times M$ diagonal matrix, which describes the CFO of the $u$ th user after the CP removal. $\widetilde{\mathbf{n}}=\mathbf{P}_{\text {rem }} \mathbf{n}$ and $\widetilde{\mathbf{x}}^{u}=\mathbf{P}_{\text {rem }} \overline{\mathbf{x}}^{u}$ are the noise and the transmitted signal after the $\mathrm{CP}$ removal, respectively. $\mathbf{H}_{C}^{u}$ is an $M \times M$ circulant matrix describing the channel of the $u$ th user.

After that, an $M$-points DFT is performed on the received signal as follows:

$$
\mathbf{R}=\mathbf{F}_{M} \mathbf{r}=\sum_{u=1}^{U} \Pi_{\mathrm{cir}}^{u} \Lambda^{u} \overline{\mathbf{X}}^{u}+\mathbf{N},
$$

where $\Pi_{\text {cir }}^{u}=\mathbf{F}_{M} \mathbf{E}^{u} \mathbf{F}_{M}^{-1}$ is a circulant matrix representing the interference from the $u$ th user. $\overline{\mathbf{X}}^{u}=\mathbf{F}_{M} \widetilde{\mathbf{X}}^{u}$ is an $M \times 1$ vector. $\mathbf{N}$ is the DFT of $\tilde{\mathbf{n}}$. The simplification of (9) depends on the fact that $\mathbf{H}_{c}^{u}=\mathbf{F}_{M}^{-1} \Lambda^{u} \mathbf{F}_{M}$, where $\Lambda^{u}$ is an $M \times M$ diagonal matrix containing the DFT of the circulant sequence of $\mathbf{H}_{C}^{u}$.

After that, the Frequency Domain Equalization (FDE), the $M$-points IDFT, and the DCT-SC-FDMA demodulation processes are performed to provide the estimate of the modulated symbols as follows:

$$
\widehat{\mathbf{x}}^{u}=\mathbf{D}_{N}^{-1} \mathbf{M}_{R}^{u} \mathbf{D}_{M} \mathbf{F}_{M}^{-1} \mathbf{W}^{u} \mathbf{R},
$$

where $\hat{\mathbf{x}}^{u}$ is an $N \times 1$ vector containing the estimate of the modulated symbols. $\mathbf{W}^{u}$ is an $M \times M$ matrix representing the FDE of the $u$ th user data. $\mathbf{M}_{R}^{u}$ is an $N \times M$ subcarriers demapping matrix of the $u$ th user. The entries of $\mathbf{M}_{R}^{u}$ for both the DCT-LFDMA and the DCT-IFDMA systems are given by taking the transpose of (2) and (3), respectively, that is, $\mathbf{M}_{R}^{u}=$ $\mathbf{M}_{T}^{u T}$.

Equation (10) can be written as follows:

$$
\hat{\mathbf{x}}^{k}=\mathbf{A}^{k} \mathbf{x}^{k}+\overline{\mathbf{A}}^{k} \mathbf{x}^{k}+\sum_{\substack{u=1 \\ u \neq k}}^{U} \mathbf{B}^{u} \mathbf{x}^{u}+\widehat{\mathbf{n}} .
$$

The structures of all components of (11) are given as follows:

$$
\begin{gathered}
\mathbf{A}^{k}=\operatorname{diag}\left(\mathbf{D}_{N}^{-1} \mathbf{M}_{R}^{k} \mathbf{D}_{M} \mathbf{F}_{M}^{-1} \mathbf{W}^{k} \Pi_{\mathrm{cir}}^{k} \Lambda^{k} \mathbf{F}_{M} \mathbf{D}_{M}^{-1} \mathbf{M}_{T}^{k} \mathbf{D}_{N}\right), \\
\overline{\mathbf{A}}^{k}=\mathbf{D}_{N}^{-1} \mathbf{M}_{R}^{k} \mathbf{D}_{M} \mathbf{F}_{M}^{-1} \mathbf{W}^{k} \boldsymbol{\Pi}_{\mathrm{cir}}^{k} \boldsymbol{\Lambda}^{k} \mathbf{F}_{M} \mathbf{D}_{M}^{-1} \mathbf{M}_{T}^{k} \mathbf{D}_{N}-\mathbf{A}^{k}, \\
\hat{\mathbf{n}}=\mathbf{D}_{N}^{-1} \mathbf{M}_{R}^{k} \mathbf{D}_{M} \mathbf{F}_{M}^{-1} \mathbf{W}^{k} \mathbf{N} \\
\mathbf{B}^{u}=\mathbf{D}_{N}^{-1} \mathbf{M}_{R}^{k} \mathbf{D}_{M} \mathbf{F}_{M}^{-1} \mathbf{W}^{k} \boldsymbol{\Pi}_{\mathrm{cir}}^{u} \boldsymbol{\Lambda}^{u} \mathbf{F}_{M} \mathbf{D}_{M}^{-1} \mathbf{M}_{T}^{u} \mathbf{D}_{N} .
\end{gathered}
$$

Finally, the demodulation and the decoding processes take place in the time domain.

\section{The Proposed Equalization Scheme}

3.1. Mathematical Model. To derive the MMSE equalization matrix of the proposed scheme, (9) must be rearranged as follows:

$$
\mathbf{R}=\Pi_{P}^{k} \overline{\mathbf{X}}^{k}+\mathbf{N}_{P}
$$

where $\boldsymbol{\Pi}_{P}^{k}=\boldsymbol{\Pi}_{\text {cir }}^{k} \Lambda^{k}$ is the $M \times M$ interference matrix of the $k$ th user. $\mathbf{N}_{P}=\sum_{u=1, u \neq k}^{U} \Pi_{P}^{u} \overline{\mathbf{X}}^{u}+\mathbf{N}$ is the MAI plus noise matrix. Now, we define the error e between the estimated symbols $\hat{\overline{\mathbf{X}}}^{k}=\mathbf{W}_{\text {proposed }}^{k} \mathbf{R}$ and the transmitted symbols $\overline{\mathbf{X}}^{k}$ as follows:

$$
\mathbf{e}^{k}=\mathbf{W}_{\text {proposed }}^{k} \mathbf{R}-\overline{\mathbf{X}}^{k} \text {. }
$$

The equalization matrix of the $k$ th user is determined by the minimization of the following Mean Square Error (MSE) cost function:

$$
\mathbf{J}^{k}=E\left\{\left\|\mathbf{e}^{k}\right\|^{2}\right\}=E\left\{\left\|\mathbf{W}_{\text {Proposed }}^{k} \mathbf{R}-\overline{\mathbf{X}}^{k}\right\|^{2}\right\},
$$

where $E\{\cdot\}$ is the expectation. Solving $\partial \mathbf{J}^{k} / \partial \mathbf{W}_{\text {proposed }}^{k}=0$, we obtain that

$$
\mathbf{W}_{\text {Proposed }}^{k}=\mathbf{R}_{X}^{k} \Pi_{P}^{k^{\mathbf{H}}}\left(\boldsymbol{\Pi}_{P}^{k} \mathbf{R}_{X}^{k} \Pi_{P}^{k^{\mathbf{H}}}+\mathbf{R}_{N_{P}}^{k}\right)^{-1},
$$

where $\mathbf{R}_{X}^{k}$ and $\mathbf{R}_{N_{P}}^{k}$ are the data and the overall noise (MAI plus noise) covariance matrices of the kth user. We assume that the noise is additive white Gaussian with zero mean and covariance $\sigma_{n}^{2}$, such that $E\left[\mathbf{N}_{d} \mathbf{N}_{d}^{H}\right]=\sigma_{n}^{2} \mathbf{I}_{N}$. Then, $\mathbf{R}_{N_{P}}^{k}$ can be obtained as follows:

$$
\begin{aligned}
\mathbf{R}_{N_{P}}^{k} & =E\left\{\left(\sum_{\substack{u=1 \\
u \neq k}}^{U} \Pi_{P}^{u} \overline{\mathbf{X}}^{k}+\mathbf{N}_{d}\right)\left(\sum_{\substack{u=1 \\
u \neq k}}^{U} \Pi_{P}^{u} \overline{\mathbf{X}}^{u}+\mathbf{N}\right)^{H}\right\} \\
& =\sum_{\substack{u=1 \\
u \neq k}}^{U} \Pi_{P}^{u} \mathbf{R}_{X}^{u} \boldsymbol{\Pi}_{P}^{u H}+\sigma_{n}^{2} \mathbf{I}_{M} .
\end{aligned}
$$




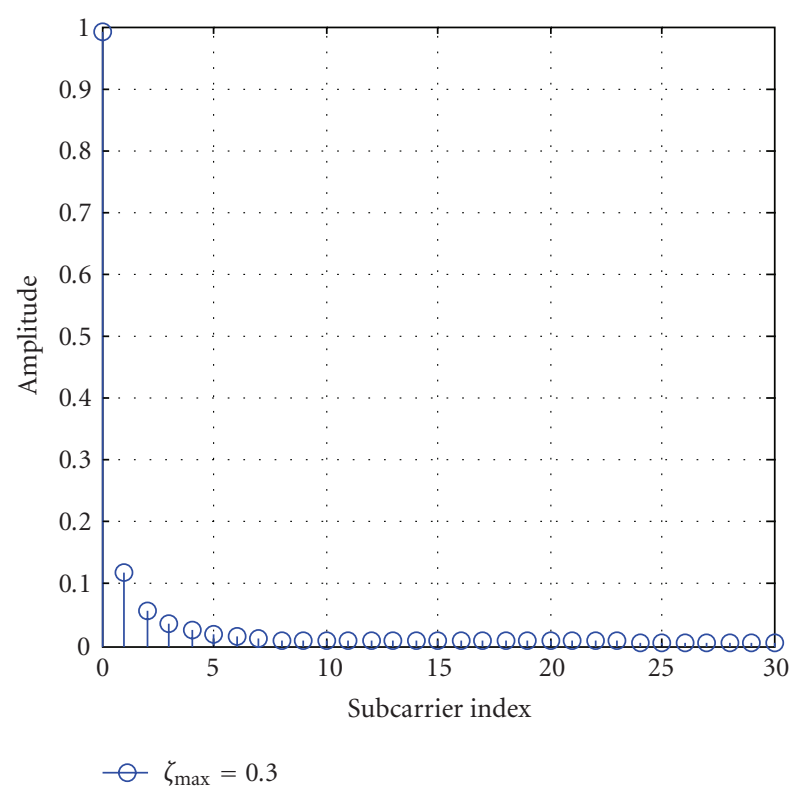

Figure 2: Amplitude versus the subcarrier index.

If all users have the same power and the average powers of the received signals on all subcarriers are the same and denoted as $\sigma_{X}^{2}$, we have $\mathbf{R}_{X}^{k}=\sigma_{X}^{2} \mathbf{I}_{M}$. As a result, (16) can be simplified as follows:

$$
\begin{aligned}
& \mathbf{W}_{\text {proposed }}^{k} \\
& \quad=\left(\boldsymbol{\Pi}_{P}^{k^{H}} \boldsymbol{\Pi}_{P}^{k}+\sum_{\substack{i=1 \\
i \neq u}}^{U} \boldsymbol{\Pi}_{P}^{u H} \boldsymbol{\Pi}_{P}^{u}+\left(\frac{1}{\mathrm{SNR}}\right) \mathbf{I}_{M}\right)^{-1} \boldsymbol{\Pi}_{P}^{k^{H}},
\end{aligned}
$$

where SNR $=\left(\sigma_{X}^{2} / \sigma_{n}^{2}\right)$. The main advantage of the proposed equalization scheme is that it minimizes the MAI and the noise. Thus, the residual MAI is lower than that in the singleuser [7] and the circular convolution [7, 10] detectors as we will see in the simulation results. In addition, the proposed scheme requires a single DFT stage for all users, similar to the circular convolution detector, since it is performed in the frequency domain.

3.2. Banded Implementation. The proposed equalization scheme is able to remove the interference for each user. However, calculating and inverting the $M \times M$ matrix is practically difficult for a large DFT size. In the proposed scheme, the complexity can be reduced, since most of the elements in $\Pi_{P}^{k}$ are zeros. Figure 2 gives plots for the amplitude of the first row of $\Pi_{P}^{k}$ in the DCT-SC-FDMA system. The subcarrier with index 0 is the desired subcarrier whereas the other subcarriers represent the interference. We consider a system with $N=32, M=128$, and $U=4$. The frequency offset is a random variable with uniform distribution in $[-0.3,0.3]$.

As shown in the figure, the amplitude of the interference caused by any subcarrier on the subcarrier 0 decreases as the frequency spacing between these subcarriers increases. As a result, a threshold $r$ for the number of subcarriers, beyond which the interference is neglected, can be introduced as a design parameter. From Figure 2, we can see that $r=10$ is the best choice to give a good performance in the proposed equalization scheme with an acceptable complexity.

3.3. Complexity Evaluation. For the proposed MMSE equalization scheme, the inversion of an $M \times M$ matrix for each user is required, which is practically difficult for a large $M$. The full system implementation requires a complexity of $O\left(M^{3}\right)$, which is large for a system with a large number of active subcarriers. However, the structure of the approximated interference matrix is a banded structure. Thus, the banded matrix implementation is considered to reduce the complexity of the proposed equalization scheme. The total number of operations required in our banded matrix implementation for all users is approximately $M U\left[16 r^{2}+26 r+5\right]$ $[13,14]$. Hence, for large values of $M$, the overall complexity is lower than that of the full system implementation.

\section{The Proposed MMSE + PIC Scheme}

It is known that the DCT-IFDMA system is more sensitive to CFOs than the DCT-LFDMA system. So, at high CFOs and SNR values, the residual MAI after the proposed MMSE equalization may degrade the Bit Error Rate (BER) performance of the DCT-IFDMA system. To solve this problem, the proposed MMSE equalizer is combined with PIC to further reduce the effect of the residual MAI on the DCT-IFDMA system, as shown in Figure 3. In the proposed MMSE + PIC scheme, the MMSE equalizer is used to estimate the MAI interference, which is then regenerated and removed from the original received signal using the PIC in the frequency domain.

\section{Simulation Results}

To evaluate the performance of the proposed schemes, some simulation experiments are carried out. We consider an uplink DCT-SC-FDMA system with 512 subcarriers. In this system, there are four users with 128 subcarriers allocated to each user. The users employ Quadrature Phase Shift Keying (QPSK) mapping for their data symbols. The channel model used for simulations is the vehicular A model [15]. A convolutional code with rate $1 / 2$, constraint length 7 , and octal generator polynomial $(133,171)$ is used. Each frequency offset is a random variable with uniform distribution in $[-0.3,0.3]$. The CFOs are chosen randomly to simulate a more practical scenario. For the comparison purpose, singleuser detector [7], and the circular convolution detector [7, 10] are simulated for the uplink DCT-SC-FDMA system.

Figure 4 shows a comparison in the BER performance between the single-user detector, the circular convolution detector, and the proposed MMSE equalization scheme for the DCT-SC-FDMA system. The DCT-SC-FDMA system without CFOs and the DCT-SC-FDMA system with CFOs but without compensation are also studied for comparison. 


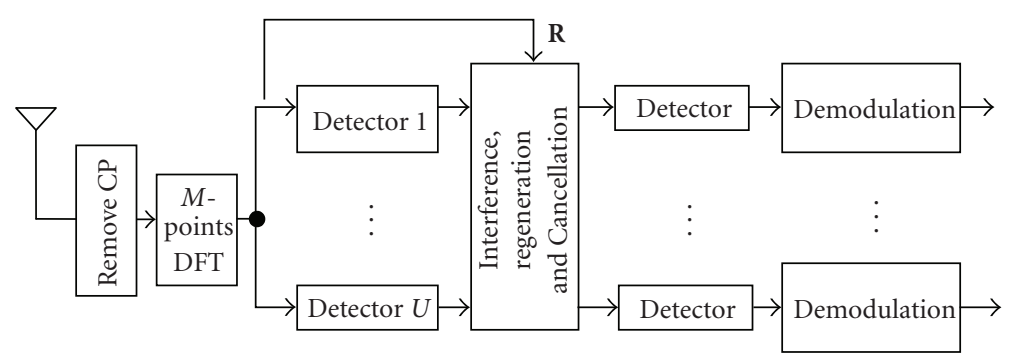

(a)

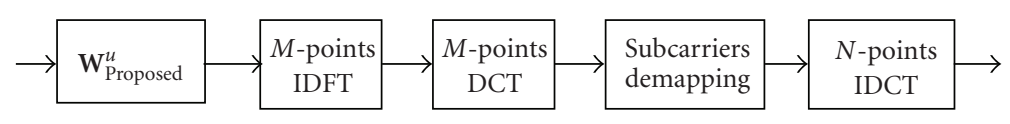

(b)

FIGURE 3: Block diagram of the proposed MMSE + PIC scheme. (a) Structure of the receiver. (b) Structure of the detector.

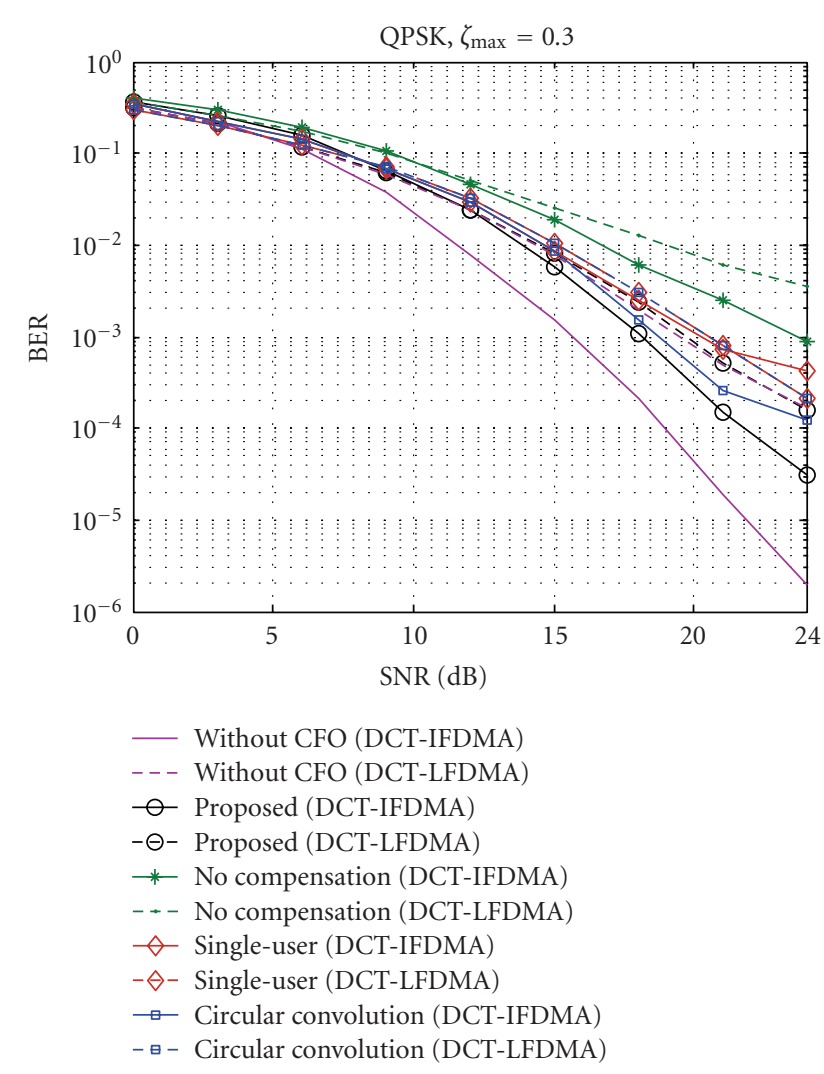

FIGURE 4: BER versus the SNR for the DCT-SC-FDMA system for all schemes.

It is clear that the proposed MMSE scheme significantly outperforms the conventional schemes, especially at high SNR values. Although the performance of the proposed scheme for the DCT-IFDMA system is superior to all conventional schemes, the performance loss is about $2.5 \mathrm{~dB}$ at a BER $=10^{-3}$. Adding a PIC stage to the proposed scheme can avoid this loss and provide a better BER performance, especially at large CFOs values.

Figure 5 shows the BER performance of the proposed MMSE and MMSE + PIC schemes for the DCT-IFDMA

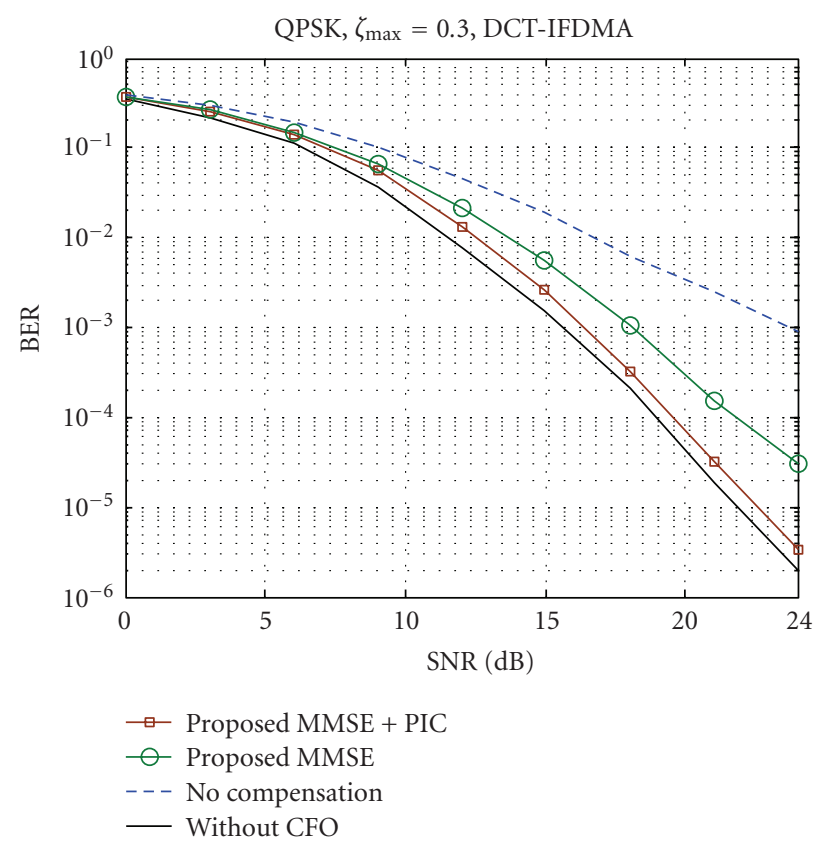

FIGURE 5: BER versus the SNR for the DCT-IFDMA system with the proposed schemes.

system. From this figure, it is clear that the PIC can avoid the MAI and provide better BER performance than the MMSE scheme. It is observed from Figure 5 that the performance loss due to the proposed MMSE + PIC is $0.5 \mathrm{~dB}$ at a BER = $10^{-3}$, which is acceptable.

Figure 6 illustrates the BER performance of the proposed schemes versus the maximum normalized $\mathrm{CFO} \zeta_{\max }$ for the DCT-IFDMA system. It can be seen that the performance of the proposed MMSE + PIC scheme is always better than the MMSE scheme, especially at large CFOs values.

The impact of the channel estimation errors on the performance of the proposed schemes for the DCT-SCFDMA system is studied and shown in Figures 7 and 8. $\mathrm{SNR}=20 \mathrm{~dB}$ is considered. The estimated CFOs are obtained by adding the true values of the CFOs to 


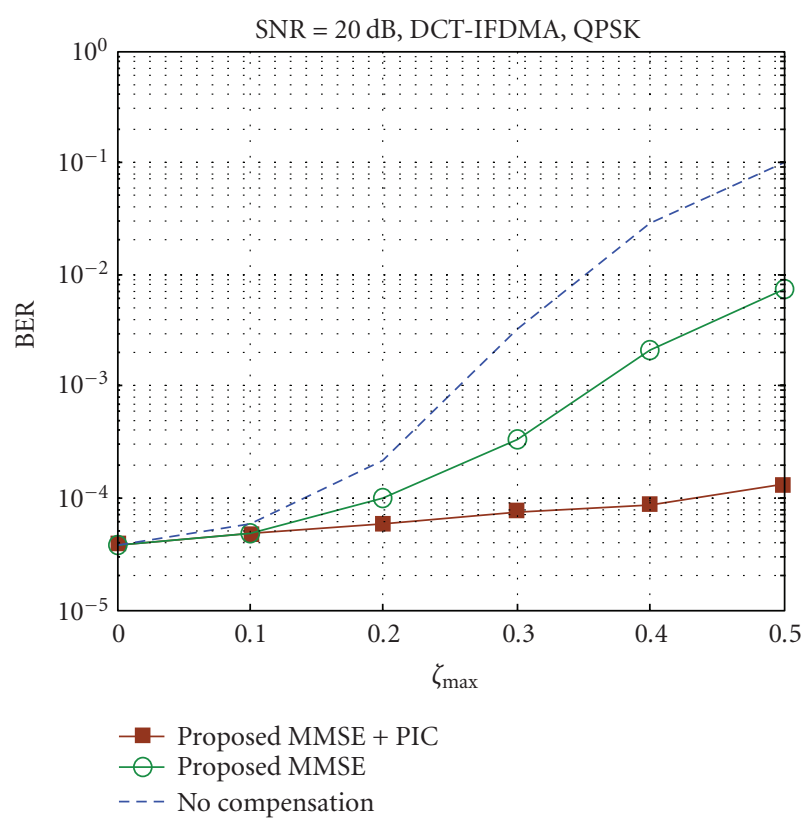

FIGURE 6: BER versus the $\zeta_{\max }$ for the DCT-IFDMA system with the proposed schemes.

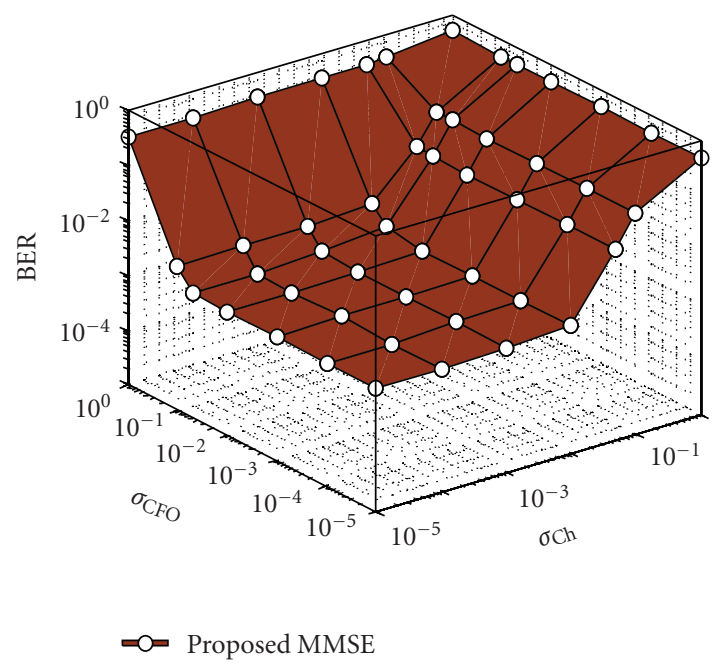

FIGURE 7: BER performance of the DCT-LFDMA system with the MMSE scheme in the presence of the estimation errors at SNR = $20 \mathrm{~dB}$.

a zero-mean independent Gaussian random variable. The estimated channels are obtained by the same manner.

Figure 7 shows that the performance of the DCT-LFDMA system with the proposed MMSE scheme starts to degrade as the standard deviation of the CFOs estimation error $\left(\delta_{\mathrm{CFO}}\right)$ becomes larger than 0.05 and the standard deviation of the channel coefficients estimation error $\left(\delta_{\mathrm{Ch}}\right)$ becomes larger than 0.01 . Figure 8 shows that the performance of the DCT-IFDMA system with the proposed MMSE and the proposed MMSE + PIC schemes starts to degrade as $\delta_{\mathrm{CFO}}$ becomes larger than 0.01 and $\delta_{\mathrm{Ch}}$ becomes larger than 0.001 .

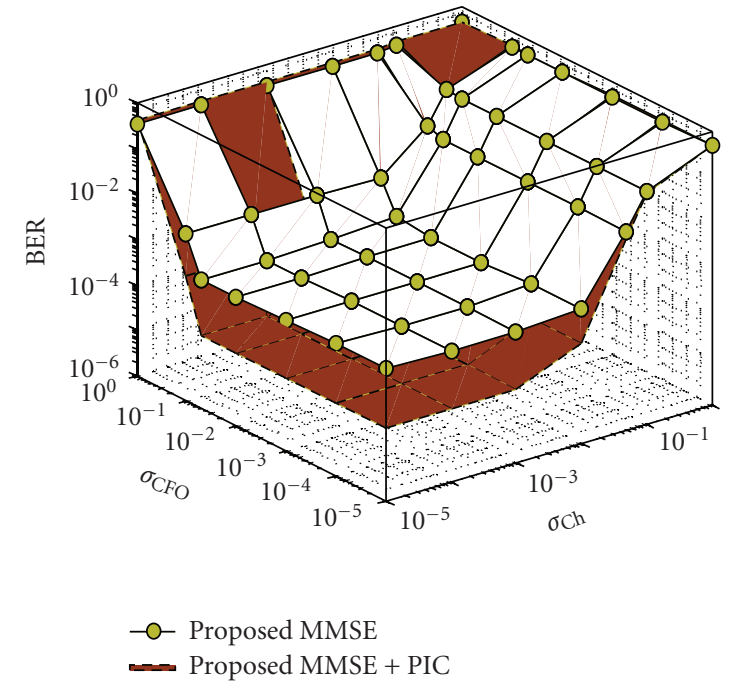

Figure 8: BER performance of the DCT-IFDMA system with the proposed MMSE and MMSE + PIC schemes in the presence of the estimation errors at $\mathrm{SNR}=20 \mathrm{~dB}$.

This indicates that the proposed compensation schemes are robust to the estimation errors.

\section{Conclusions}

In this paper, the issue of the CFOs in the uplink DCT-SCFDMA system is investigated and compensated. Simulation results show that CFOs destroy the orthogonality of the subcarriers and result in ICI and MAI, which degrades the BER performance. This paper presented two new compensation schemes. Simulation results show that the proposed MMSE and MMSE + PIC schemes are able to mitigate the impact of CFOs to provide a better BER performance for DCT-SCFDMA system. Also, simulation results demonstrate that the proposed MMSE and MMSE + PIC schemes outperform both the circular convolution detector and the single-user detector. Moreover, it is found that the proposed MMSE and MMSE + PIC schemes are robust to the estimation errors.

\section{References}

[1] H. G. Myung, J. Lim, and D. J. Goodman, "Single carrier FDMA for uplink wireless transmission," IEEE Vehicular Technology Magazine, vol. 1, no. 3, pp. 30-38, 2006.

[2] F. E. Abd El-Samie, F. S. Al-Kamali, M. I. Dessouky, B. M. Sallam, and F. Shawki, "Performance enhancement of SCFDMA systems using a companding technique," Annales des Telecommunications/Annals of Telecommunications, vol. 65, no. 5-6, pp. 293-300, 2010.

[3] A. Wilzeck, Q. Cai, M. Schiewer, and T. Kaiser, "Effect of multiple carrier frequency offsets in MIMO SC-FDMA systems," in Proceedings of the International ITG/IEEE Workshop on Smart Antennas, Vienna, Austria, February 2007.

[4] H. Cheon, "Frequency offset estimation for high speed users in E-UTRA uplink," in Proceedings of the 18th Annual IEEE International Symposium on Personal, Indoor and Mobile Radio Communications (PIMRC '07), September 2007. 
[5] F. S. Al-Kamali, M. I. Dessouky, B. M. Sallam, F. Shawki, and F. E. Abd El-Samie, "A new single carrier FDMA system based on the discrete cosine transform," in Proceedings of the International Conference on Computer Engineering and Systems (ICCES '09), pp. 555-560, 2009.

[6] J. V. Beek, P. O. Börjesson, M.-L. Boucheret et al., "A time and frequency synchronization scheme for multiuser OFDM," IEEE Journal on Selected Areas in Communications, vol. 17, no. 11, pp. 1900-1914, 1999.

[7] J. Choi, C. Lee, H. W. Jung, and Y. H. Lee, "Carrier frequency offset compensation for uplink of OFDM-FDMA systems," IEEE Communications Letters, vol. 4, no. 12, pp. 414-416, 2000.

[8] K. Sathananthan, R. M. A. P. Rajatheva, and S. B. Slimane, "Cancellation technique to reduce intercarrier interference in OFDM," Electronics Letters, vol. 36, no. 25, pp. 2078-2079, 2000.

[9] K. Sathananthan and C. Tellambura, "Partial transmit sequence and selected mapping schemes to reduce ICI in OFDM systems," IEEE Communications Letters, vol. 6, no. 8, pp. 313-315, 2002.

[10] D. Huang and K. B. Letaief, "An interference-cancellation scheme for carrier frequency offsets correction in OFDMA systems," IEEE Transactions on Communications, vol. 53, no. 7, pp. 1155-1165, 2005.

[11] D. Yan, W. Bai, Y. Xiao, and S. Li, "Multiuser interference suppression for uplink interleaved FDMA with carrier frequency offset," in Proceedings of the International Conference on Wireless Communications and Signal Processing (WCSP'09), 2009.

[12] X. Zhang, H.-G. Ryu, and Y. Li, "Joint suppression of phase noise and CFO by block type pilots," in Proccedings of the 9th International Symposium on Communications and Information Technology (ISCIT '09), pp. 466-469, 2009.

[13] Z. Cao, U. Tureli, and Y.-D. Yao, "Low-complexity orthogonal spectral signal construction for generalized OFDMA uplink with frequency synchronization errors," IEEE Transactions on Vehicular Technology, vol. 56, no. 3, pp. 1143-1154, 2007.

[14] G. H. Golub and C. F. Van Loan, Matrix Computations, The Johns Hopkins University Press, 3rd edition, 1996.

[15] 3rd Generation Partnership Project and 3GPP TS 25.101, "Technical Specification Group Radio Access Network; User Equipment (UE) Radio Transmission and Reception (FDD) (Release 7), Section B.2.2," September 2007. 

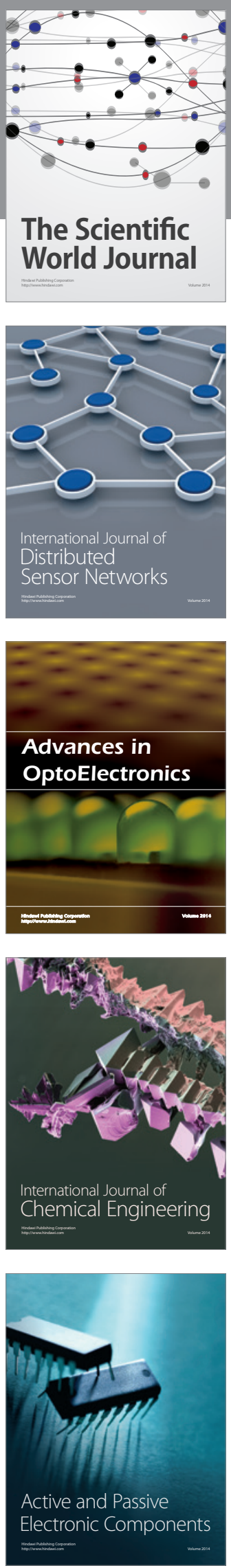
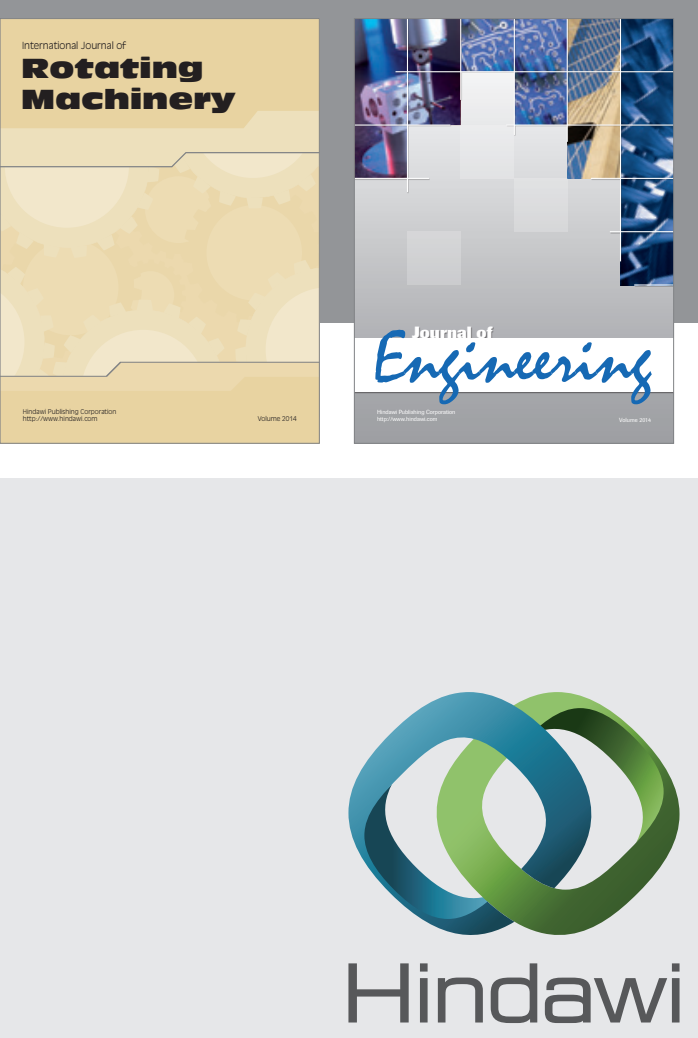

Submit your manuscripts at

http://www.hindawi.com
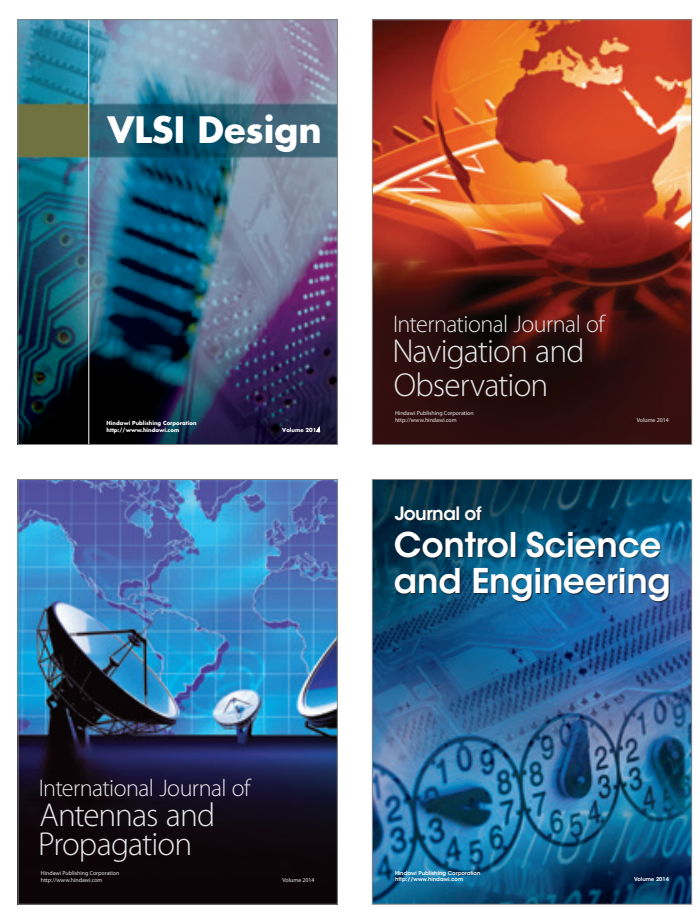
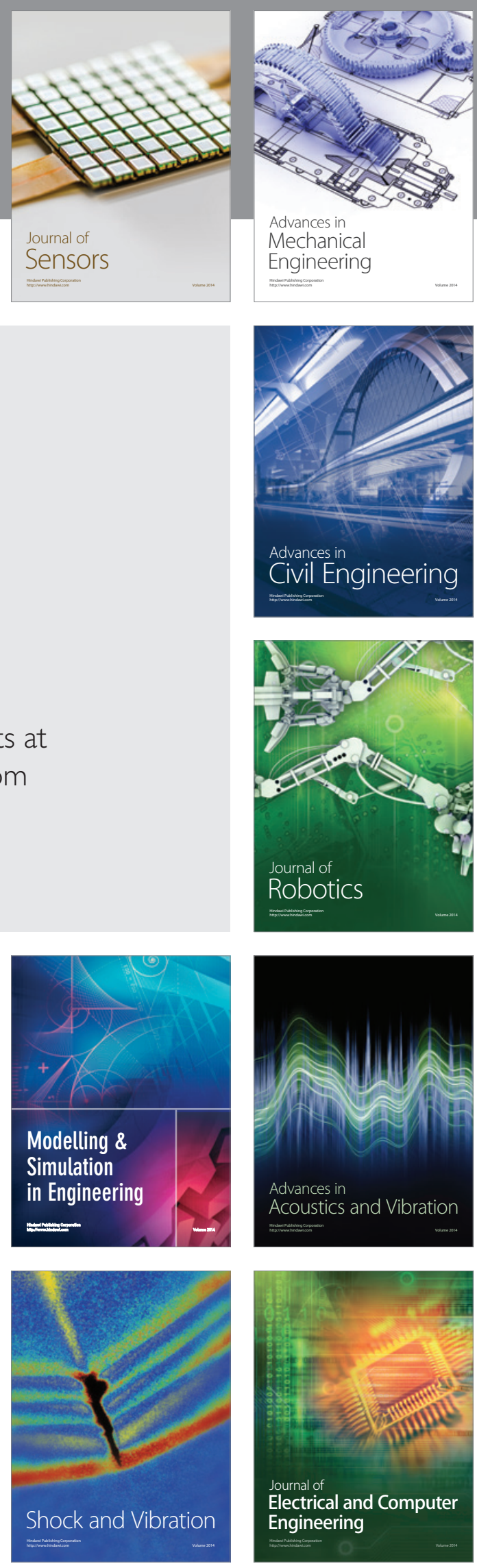